\title{
Correction to: A superficial femoral artery calcification: insights \\ from a multi-imaging modality assessment with computed tomography, electronic high-resolution angioscopy, and optical frequency domain imaging
}

Yuji Nishimoto $^{1}{ }^{1} \cdot$ Rei Fukuhara $^{1} \cdot$ Fumiyuki Otsuka ${ }^{2} \cdot$ Masanao Toma $^{1} \cdot$ Tadashi Miyamoto $^{1} \cdot$ Yukihito Sato $^{1}$

Published online: 18 July 2019

(c) Japanese Association of Cardiovascular Intervention and Therapeutics 2019

\section{Correction to: \\ Cardiovascular Intervention and Therapeutics \\ https://doi.org/10.1007/s12928-019-00601-0}

In the original publication of the article, the first author name "Yuji Nishimoto" was repeated in the author group.

The duplicate author name is removed in this correction.

The original article has been corrected.

The original article can be found online at https://doi.org/10.1007/ s12928-019-00601-0.

Yuji Nishimoto

yuji.nishimoto@gmail.com

1 Department of Cardiology, Hyogo Prefectural Amagasaki

General Medical Center, 2-17-77 Higashinaniwa-cho,

Amagasaki 660-8550, Japan

2 Department of Cardiovascular Medicine, National Cerebral and Cardiovascular Center, Osaka, Japan 